\title{
Immunological Responses against Different Lineages of Influenza B Antigen in School Children during Two Consecutive Seasons
}

\author{
Day-Yu Chao ${ }^{* \#}$, Kuang-Fu Cheng ${ }^{2 *}$, Yu-Hsin Liao ${ }^{1}$, Ming-Tsan Liu ${ }^{3}$, Ying-Hen Hsieh ${ }^{4,5}$, \\ Tsai-Chung $\mathrm{Li}^{2,4}$, Trong-Neng $\mathrm{Wu}^{4,5}$, Chiu-Ying Chen ${ }^{5}$ \\ ${ }^{1}$ Graduate Institute of Microbiology and Public Health, College of Veterinary Medicine, National Chung-Hsing \\ University, Taichung, Taiwan \\ ${ }^{2} \mathrm{CMU}$ Biostatistics Center, China Medical University, Taichung, Taiwan \\ ${ }^{3}$ National Influenza Center, Center for Disease Control, Taipei, Taiwan \\ ${ }^{4}$ Graduate Institute of Biostatistics, China Medical University, Taichung, Taiwan \\ ${ }^{5}$ Department of Public Health, China Medical University, Taichung, Taiwan \\ Email: ${ }^{\#}$ dychao@nchu.edu.tw
}

Received 25 September 2014; revised 14 November 2014; accepted 27 November 2014

Copyright (C) 2014 by authors and Scientific Research Publishing Inc.

This work is licensed under the Creative Commons Attribution International License (CC BY).

http://creativecommons.org/licenses/by/4.0/

(c) (i) Open Access

\begin{abstract}
While Influenza B viruses currently circulating worldwide are of two distinct evolutionary hemagglutinin lineages, current trivalent inactivated influenza virus vaccines (TIV) contain only a single component. Single doses of TIV containing B antigen of B/Florida/4/2006 (Yamagata-like) or $B /$ Brisbane/60/2008 (Victoria-like) were administered during 2008/2009 and 2009/2010 influenza seasons, respectively. The objective of this study was to evaluate the immunological response against different lineages of $B$ antigens in school-aged children. A non-randomized seroepidemiological study was conducted and the immunogenicity responses based on sero-protection rate and geometric mean titre ratio (GMTR) of hemagglutination inhibition (HI) antibodies were measured before and after immunization as well as post-influenza season. Our results suggested that school-aged children under the age of 9 years receiving TIV vaccination induced and retained higher level of sero-protection rate $(66.7 \%$ and $69 \%$ for the $2008-09$ and $2009-10$ season, respectively) to the homologous lineage than the heterologous lineage post-vaccination $(19.4 \%$ and $27.6 \%$ for the 2008-09 and 2009-10 season, respectively). The need for the quadrivalent TIV by including both lineages of influenza $B$ viruses is recommended in this study, particularly for children under the age of 9 years.
\end{abstract}

\footnotetext{
*These authors contributed equally to this work as first authors.

${ }^{\#}$ Corresponding author.
}

How to cite this paper: Chao, D.-Y., Cheng, K.-F., Liao, Y.-H., Liu, M.-T., Hsieh, Y.-H., Li, T.-C., Wu, T.-N. and Chen, C.-Y. (2014) Immunological Responses against Different Lineages of Influenza B Antigen in School Children during Two Consecutive Seasons. Health, 6, 2837-2847. http://dx.doi.org/10.4236/health.2014.621322 
Keywords

Children, Immunology, Influenza, Trivalent Inactivated Vaccine (TIV), Yamagata/Victoria Lineage

\section{Introduction}

Influenza viruses cause annual epidemics and occasional pandemics of acute respiratory disease in human populations leading to significant health threats worldwide [1]. Vaccination is the primary intervention method for preventing clinical disease and associated complications [2] [3]. The virus is capable of evading immune recognition through continual antigenic drift of its surface glycoproteins, hemagglutinin (HA) and neuraminidase (NA), resulting in a loss of immunity and vaccine mismatch. As a consequence, monitoring antigenic and genetic variations of circulating influenza viruses are crucial for the selection of annual strains [4].

While two types of influenza A viruses (H3N2 and H1N1) have been in circulation since 1968 and 1977 respectively, two distinct evolutionary lines of the influenza B virus represented in human populations by the reference strains B/Victoria/2/87 and B/Yamagata/16/88 have gained attention since 2001/2002 [5]. Influenza virological surveillance evidenced the co-circulation of the two lineages of the influenza $\mathrm{B}$ virus during the same season in many parts of the world, including Europe, Italy and Taiwan [6]-[8]. Since Asia has been considered the gene pool for influenza viruses, the Taiwan Centers for Disease Control (Taiwan-CDC) have been coordinating a laboratory-based virological surveillance network since 2000 to provide updated information about viral characteristics and activities [9] [10]. After the co-circulation of B/Victoria/2/87- and B/Yamagata/16/88-like viruses in the 2004-2005 influenza season in Taiwan, both lineages circulated with alternating presence, and new genetic clades with amino acid substitutions appeared [11] [12].

Current trivalent inactivated influenza virus vaccines (TIV) contain only a single influenza B virus component, either a Yamagata-like (influenza season 2004/2005 and 2005/2006) or a Victoria-like (influenza season 2003/ 2004, 2006/2007 and 2007/2008) strain [13]. The selection was based on epidemiological data on the geographic advance of infections, and antigenic and genetic results of recently and most frequently isolated B viruses. These two lineages are distinct antigenically, raising the concern that re-immunization might be required when antigenically altered strains appear [14]-[16]. The current recommendation for TIV vaccination in previously unvaccinated children under the age of 9 years is to receive 2 doses of TIV when vaccinated for the first time and 1 dose annually thereafter. This recommendation assumes effective prime-boosting across related antigens of the 2 influenza A/subtypes, but the declining number of children receiving the second dose 4 weeks later after the first dose makes it logistically challenging.

Since 2008, the Advisory Committee on Immunization Practices (ACIP) recommends annual influenza vaccination for all children of 6 months to 18 years, including all school-aged children [17]. High vaccination coverage of school-aged children is an important public health objective because of the direct benefits to immunized children and the potential indirect protection by reducing student and teacher absenteeism, allowing schools to better accomplish their primary mission and reducing morbidity/mortality for the broader community [18]-[20]. In Taiwan, the national free pediatric immunization programme of TIV has been implemented since 2007, and all children under the age of 9 years from the elementary schools will receive one dose of TIV annually. So far, limited study has been conducted to understand the immunogenicity after receiving different lineages of B antigens in school-aged children less than the age of 9 years - the group considered the primary community spreader of influenza viruses [18] [21]. Most among them are not naïve to influenza viruses and have antibodies against B of both lineages [21] [22]. The current non-randomized sero-epidemiological study conducting during 2008-2010 influenza seasons provided a good opportunity to evaluate the immunological response in schoolaged children who received two antigenic-distinct influenza B virus lineages included in trivalent inactivated influenza vaccine (TIV) in two consecutive seasons.

In order to determine whether immunization with a B viral antigen of one lineage is sufficient to produce antibodies with cross-reactivity against both lineages, we analyzed the haemagglutination inhibition (HI) antibody response of school-aged children who received different B components of TIV in the 2008/09 and 2009/10 influenza seasons. Immunogenicity was measured for local strains of influenza B viruses of the B/Victoria lineage (B/Taiwan/5908/2009) and of the B/Yamagata lineage (B/Taiwan/29/2008) circulating in the community before 
and after vaccination. In the current study, we aimed to discover the immunological responses of children immunized with B viral antigens of one lineage, and subsequently with the other in the following influenza season, as well as the antibody protection during winter epidemics in Taiwan.

\section{Materials and Methods}

\subsection{Subject Enrollment and Serological Specimens}

Since the year 2007, the government in Taiwan has implemented a free vaccination programme through which all children in elementary schools receive a free trivalent influenza vaccination (TIV) annually. A single dose of commercially-available AdimFlu-S (Adimmune Corporation, Taiwan) contains 0.5-mL of 15 ug HA of the H1N1, H3N2 and B antigens from the designated year. A non-randomized sero-epidemiological study was conducted by the Center for Infectious Disease Education and Research (CIDER) influenza research group at China Medical University (CMU) to investigate household transmission and vaccine effectiveness. Schoolchildren 6 - 12 years of age, located in urban (Taichung city) and rural (Nantou county) areas in central Taiwan, were recruited and their serum samples were taken by trained nurses during five sampling periods in two consecutive seasons, 2008-2009 and 2009-2010, which are referred to as 2008/09 post-vaccination (sera collected between November 2008 and January 2009), 2008/09 post-season (sera collected between April and June of 2009), 2009/10 pre-vaccination (sera collected between September and October of 2009), 2009/10 post-vaccination (sera collected between November 2009 and January 2010) and 2009/10 post-season (samples collected between April and June of 2010) in this study.

The study protocol based on laboratory data was established to include the paired sera drawn from the study subjects from any two consecutive time periods to define the immune response before and after vaccination or wild-type virus infection. The duration between the date of vaccination and post-vaccination sera collected was in the range of 30 - 150 days (median, 75 days). Two questionnaire interviews were also conducted by trained interviewers regarding basic demographic and social contact information, in addition to bi-weekly telephone interviews recording upper respiratory-related symptoms during the influenza seasons. The subjects without clear information on vaccination status or the duration shorter than 4 weeks between two blood samples drawn before and after vaccination will be excluded for further analysis. All subjects gave informed consent and the study was approved by the Medical Ethics Committee of China Medical University (DMR96-IRB-216).

\subsection{Laboratory Assay}

Antibody titres were measured by a hemagglutination inhibition (HI) assay following the standard protocol by the World Health Organization (WHO) [23] [24]. The B/Florida/4/2006-like (B/Yamagata lineage) and B/Brisbane/60/2008-like (B/Victoria lineage) vaccine strain B virus obtained from Adimmune (Taichung, Taiwan) was used to evaluate the immune response before and after TIV vaccination in children. The wild-type virus strains representing the genetically and antigenically drifted B viruses, referred to as B/Taiwan/5908/2009 (B/Victoria lineage, the heterologous and homologous wild-type strain during the 2008-09 and 2009-10 season, respectively) and B/Taiwan/29/2008 (B/Yamagata lineage, the homologous and heterologous wild-type strain during 2008-09 and 2009-10 season, respectively), were obtained from Taiwan-CDC. All viruses used in this study were cultured from Madin-Darby canine kidney (MDCK) cells and centrifuged at $1600 \mathrm{rpm}, 4^{\circ} \mathrm{C}$ to remove cell debris. For the HI assay, serum samples were pre-treated with receptor destroying enzyme and titrated in two-fold dilutions in phosphate-buffered saline (PBS) with an initial dilution of 1:10. Titres were expressed as the reciprocal of the highest dilution of serum where hemagglutination was prevented. Samples that were below the detection limit $(1: 10)$ by $\mathrm{HI}$ were assigned a titre of $1: 5$ for computational purposes in obtaining a four-fold increase and geometric mean titre (GMT) of HI titres. The seroprotection was defined as an HI titre of 1:40 or more. The GMT ratio (GMTR) was calculated by dividing the post-vaccination GMT by the pre-vaccination baseline GMT.

\subsection{Virological Surveillance}

The influenza surveillance network coordinated by Taiwan-CDC covers approximately $75 \%$ of the basic administrative units of Taiwan (cities or districts) and routinely collects clinical specimens throughout the years. Throat or nasal swabs from patients with influenza-like illnesses were collected and transported to the laborato- 
ries of the influenza surveillance network in Taiwan for analysis. On arrival, they were suspended in $1.5 \mathrm{ml}$ of serum-free DMEM medium and filtered through a 0.45 um filter. RNA extraction and subsequent real-time RT-PCR were performed together with virus culture as previously described [25] [26]. This surveillance is performed in an unbiased manner and can provide a detailed record of the timing of the introduction and evolution of a newly emerging variant.

\subsection{Statistical Analysis}

The immunogenicity outcomes, including seroprotection rate and GMTR, were evaluated based on HI titres. The point estimates and 95\% confidence intervals (CI) of the immunogenicity outcomes were calculated using a generalized linear model (GLM). The comparisons were made between the vaccination group (yes vs. no), age group (children $<9$ vs. $\geq 9$ ), and different lineage group of influenza B viruses (homologous vs. heterologous). For groups with small numbers of subjects, the non-parametric Wilcoxon rank method was calculated for immunogenicity statistics. A p-value of less than 0.05 represented statistical significance. All statistical analyses were performed using SAS statistical software version 9.2 (SAS Institute, Cary, NC).

\section{Results}

\subsection{Study Subjects}

Although the current vaccine recommendation for children less than 9 years of age is to receive 2 doses of TIV, the school children in our study under the coverage of free TIV vaccination programme implemented in Taiwan received only one dose of TIV during the study period. All subjects were divided into two groups: $<9$ and $\geq 9$ years old to evaluate the differences in immunological responses before and after vaccination. Basic demographic data, including age, gender, residential area and vaccination status of the 148 and 198 school-aged children from two consecutive seasons were described in Table 1. The median ages for these children were 8 and 11 years. The gender distribution was about the same in two consecutive seasons, except a slightly higher number of female were enrolled in 2009/10 season. In both the 2008-09 and 2009-10 seasons, a higher proportion of subjects were from rural areas than from urban cities. Furthermore, $76.2 \%$ of subjects less than 9 years of age received TIV during the 2008-09 season, but only 36.7\% were vaccinated during the 2009-10 season. In contrast, only $42.4 \%$ and $31.9 \%$ of children equal to or older than 9 years received TIV during two consecutive seasons in 2008-10. A lower proportion of children less than 9 years of age (42.9\% and 20.3\% from 2008-09 and 2009-10 seasons, respectively) reported receiving TIV from the previous influenza season. Similarly, a lower proportion receiving TIV from the previous season was also observed in children $\geq 9$ years (28.2\% and $30.5 \%$ from 2008-09 and 2009-10 seasons, respectively).

\subsection{Virological Surveillance}

As shown in Figure 1 from 2004 to 2010, influenza B was only predominant in the winter influenza seasons in 2004-05 and 2006-07 in Taiwan. After that, the influenza B virus seemed to circulate during the summer seasons in 2008 and 2010. Furthermore, an alternating pattern of different lineages was observed after 2005, with the B/Victoria lineage dominant in the 2006-07 season followed by B/Yamagata dominance in 2008, which was later replaced by the B/Victoria again in 2010.

\subsection{HI Antibody Post-Vaccination}

Since the vaccine strain of B component contained the B/Yamagata lineage (B/Florida/4/2006) in the 2008-09 influenza season and was changed to B/Victoria lineage (B/Brisbane/60/2008) in the 2009-10 season according to WHO's announcement, HI antibodies were titred against two different lineages of influenza B viruses. The proportion of sero-protection of the TIV containing a B antigen were examined in both groups of children, aged $<9$ and $\geq 9$ years, by comparing HI titres after vaccination. The results were showed in Table 2. During the 2008-09 season, the sero-protection rate (66.7\%) post-vaccination in vaccinated subjects less than 9 years was significantly higher for the vaccine strain than that (19.4\%) for the heterologous wild-type strain from different lineage, with statistical significance $(\mathrm{p}<0.05)$. A similar result was also observed in children $\geq 9$ years old after receiving TIV vaccinations. 
Table 1. Demographic characteristics of the subjects.

\begin{tabular}{|c|c|c|}
\hline & 08/09 Influenza season & 09/10 Influenza season \\
\hline school aged children aged $\leq 9$ & $N=63$ & $\mathrm{~N}=79$ \\
\hline \multicolumn{3}{|l|}{ Age (years) } \\
\hline mean \pm SD & $7.87 \pm 0.87$ & $8.26 \pm 0.78$ \\
\hline median (Q1 - Q3) & $8(7-9)$ & $8(8-9)$ \\
\hline Range & $6-9$ & $6-9$ \\
\hline \multicolumn{3}{|l|}{ Gender (\%) } \\
\hline Male & $35(55.6 \%)$ & 39 (49.4\%) \\
\hline Female & $28(44.4 \%)$ & $40(50.6 \%)$ \\
\hline \multicolumn{3}{|l|}{ Residence (\%) } \\
\hline Urban & $22(34.9 \%)$ & $24(30.4 \%)$ \\
\hline Rural & $41(65.1 \%)$ & $55(69.6 \%)$ \\
\hline \multicolumn{3}{|l|}{ Received vaccine in this year (\%) } \\
\hline Yes & $48(76.2 \%)$ & $29(36.7 \%)$ \\
\hline No & $15(23.8 \%)$ & $50(63.3 \%)$ \\
\hline \multicolumn{3}{|l|}{ Received vaccine in previous year (\%) } \\
\hline Yes & 27 (42.9\%) & $16(20.3 \%)$ \\
\hline No & $36(57.1 \%)$ & $63(79.7 \%)$ \\
\hline school aged children aged $>9$ & $\mathrm{~N}=85$ & $\mathrm{~N}=119$ \\
\hline \multicolumn{3}{|l|}{ Age (years) } \\
\hline mean $\pm \mathrm{SD}$ & $11.24 \pm 1.1$ & $11.05 \pm 0.97$ \\
\hline median (Q1 - Q3) & $11(10-12)$ & $11(10-12)$ \\
\hline Range & $10-13$ & $10-13$ \\
\hline \multicolumn{3}{|l|}{ Gender (\%) } \\
\hline Male & $40(47.1 \%)$ & $51(42.9 \%)$ \\
\hline Female & 45 (52.9\%) & $68(57.1 \%)$ \\
\hline \multicolumn{3}{|l|}{ Residence (\%) } \\
\hline Urban & 35 (41.2\%) & $41(34.5 \%)$ \\
\hline Rural & $50(58.8 \%)$ & $78(65.5 \%)$ \\
\hline \multicolumn{3}{|l|}{ Received vaccine in this year (\%) } \\
\hline Yes & $36(42.4 \%)$ & 38 (31.9\%) \\
\hline No & $49(57.6 \%)$ & $81(68.1 \%)$ \\
\hline \multicolumn{3}{|l|}{ Received vaccine in previous year (\%) } \\
\hline Yes & $24(28.2 \%)$ & $36(30.5 \%)$ \\
\hline No & 61(71.8\%) & 83 (69.5\%) \\
\hline
\end{tabular}

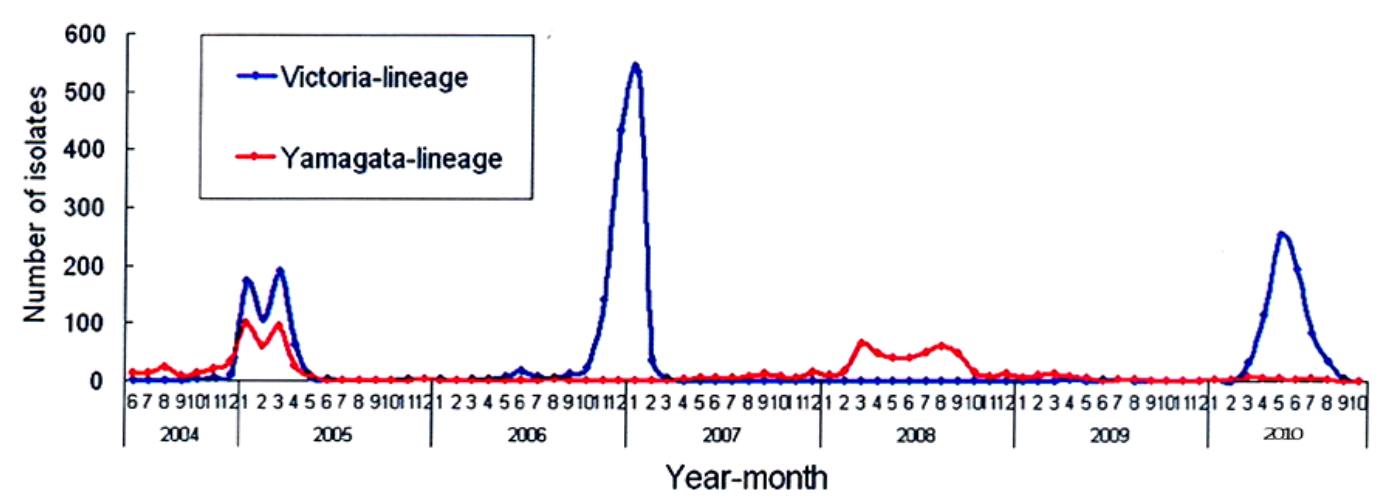

Figure 1. Genotyping results of influenza B viruses from June 2004 to October 2010 based on the influenza surveillance network in Taiwan. 
Table 2. Immune responses against different lineages of influenza B viruses in school-aged children ( $<9$ vs. $\geq 9$ years old) during 2008/09 and 2009/10 influenza season.

\begin{tabular}{|c|c|c|c|c|c|c|c|c|c|}
\hline \multicolumn{5}{|c|}{ 2008-2009 season } & \multicolumn{5}{|c|}{ 2009-2010 season } \\
\hline $\begin{array}{l}\text { Age group, In } \\
\text { Antigen }\end{array}$ & Immunization & $\begin{array}{l}\text { No. of } \\
\text { subject }\end{array}$ & \multicolumn{2}{|c|}{$\begin{array}{c}\text { Seroprotection rate, } \\
\%(95 \% \text { CI })\end{array}$} & \multirow[t]{2}{*}{ Immunization } & \multirow[t]{2}{*}{$\begin{array}{l}\text { No. of } \\
\text { subject }\end{array}$} & \multicolumn{3}{|c|}{ Seroprotection rate, \% (95\% CI) } \\
\hline \multicolumn{3}{|c|}{ School-aged children aged $<9$} & $\begin{array}{c}\text { After } \\
\text { vaccination }^{\mathrm{b}}\end{array}$ & Post-season $^{c}$ & & & $\begin{array}{c}\text { Before } \\
\text { vaccination }^{\mathrm{a}}\end{array}$ & $\begin{array}{c}\text { After } \\
\text { vaccination }\end{array}$ & Post-season $^{c}$ \\
\hline \multirow[t]{4}{*}{ Vaccine strain $^{d}$} & Yes & 48 & $66.7^{* \dagger}$ & $39.6^{\dagger}$ & Yes & 29 & $44.8^{*}$ & $69.0^{\dagger}$ & 62.1 \\
\hline & & & $(52.1-79.2)$ & $(25.0-54.2)$ & & & $(27.6-62.1)$ & $(51.7-86.2)$ & $(44.8-75.9)$ \\
\hline & No & 15 & $20^{*}$ & 26.7 & No & 50 & $82^{*}$ & $62.0^{\ddagger}$ & 46.0 \\
\hline & & & $(0-40)$ & $(6.7-46.7)$ & & & $(72.0-92.0)$ & $(48.0-75.9)$ & $(32.0-60.0)$ \\
\hline \multirow[t]{4}{*}{$\begin{array}{l}\text { Homologous } \\
\text { lineage strain }\end{array}$} & Yes & 48 & 22.9 & 8.3 & Yes & 29 & 41.4 & 44.8 & 58.6 \\
\hline & & & $(12.5-35.4)$ & $(2.1-16.7)$ & & & $(24.1-58.6)$ & $(27.6-65.4)$ & $(41.4-75.9)$ \\
\hline & No & 15 & 6.7 & 13.3 & No & 50 & 62.0 & 38.0 & 30.0 \\
\hline & & & $(0-20)$ & $(0-33.3)$ & & & $(48.0-74.0)$ & $(24.0-52.0)$ & $(18.0-44.0)$ \\
\hline \multirow[t]{4}{*}{$\begin{array}{l}\text { Heterologous } \\
\text { lineage strain }\end{array}$} & Yes & 48 & $19.4^{* \dagger}$ & $11.1^{\dagger}$ & Yes & 29 & 27.6 & $27.6^{\dagger}$ & 51.7 \\
\hline & & & $(8.3-33.3)$ & $(2.8-22.2)$ & & & $(10.3-44.8)$ & $(10.3-44.8)$ & $(34.5-69.0)$ \\
\hline & No & 15 & $0^{*}$ & 14.3 & No & 50 & 28.0 & $30.0^{\ddagger}$ & 26.0 \\
\hline & & & $(0-0)$ & $(0-42.9)$ & & & $(16.0-40.0)$ & $(18.0-44.0)$ & $(14.0-38.0)$ \\
\hline \multicolumn{10}{|c|}{ School-aged children aged $\geq 9$} \\
\hline \multirow[t]{4}{*}{ Vaccine strain $^{\mathrm{d}}$} & Yes & 36 & $72.2^{* \dagger}$ & $44.4^{\dagger}$ & Yes & 38 & 52.6 & 60.5 & 42.1 \\
\hline & & & $(56.0-86.1)$ & $(27.8-58.3)$ & & & $(34.2-68.4)$ & $(44.7-73.7)$ & $(23.7-57.9)$ \\
\hline & No & 49 & $38.8^{*}$ & 32.7 & No & 81 & 50.6 & 65.4 & 35.8 \\
\hline & & & $(26.5-51.0)$ & $(21.1-46.2)$ & & & $(38.3-59.3)$ & $(54.3-74.1)$ & $(25.9-45.7)$ \\
\hline \multirow[t]{4}{*}{$\begin{array}{l}\text { Homologous } \\
\text { lineage strain }\end{array}$} & Yes & 36 & 52.8 & 33.3 & Yes & 38 & 34.2 & 60.5 & 31.6 \\
\hline & & & $(36.6-66.7)$ & $(19.9-47.2)$ & & & $(18.4-52.6)$ & $(44.7-78.9)$ & $(18.4-44.7)$ \\
\hline & No & 49 & 24.5 & 16.3 & No & 81 & 43.2 & 49.4 & 24.7 \\
\hline & & & $(10.6$ - 38.1) & $(8.5-26.5)$ & & & (32.1 - 54.3) & $(38.3-61.7)$ & $(14.8-33.3)$ \\
\hline \multirow[t]{4}{*}{$\begin{array}{l}\text { Heterologous } \\
\text { lineage strain }\end{array}$} & Yes & 36 & $14.8^{\dagger}$ & $3.7^{\dagger}$ & Yes & 38 & 36.8 & 39.5 & 39.5 \\
\hline & & & $(3.7-33.3)$ & $(0-14.2)$ & & & $(23.7-50.0)$ & $(23.7-60.5)$ & $(23.7-55.3)$ \\
\hline & No & 49 & 2.9 & 5.9 & No & 81 & 28.4 & 39.5 & 35.8 \\
\hline & & & $(0-8.8)$ & $(0-17.1)$ & & & $(19.8-38.3)$ & $(29.6-50.6)$ & $(25.9-45.7)$ \\
\hline
\end{tabular}

*Significant different $(\mathrm{p}<0.05)$ between vaccinated and un-vaccinated subjects; ${ }^{\dagger}$ Significant different $(\mathrm{p}<0.05)$ between two lineages of Influenza B virus in vaccinated children; ${ }^{\ddagger}$ Significant different $(\mathrm{p}<0.05)$ between two lineages of Influenza B virus in un-vaccinated children. a. Sample collected from Sep. to Oct. 2009 during 2009-2010 season; b. Sample collected between Nov. 2008 and Jan. 2009 during 2008-2009 season and from Nov. 2009 to Jan. 2010 during 2009-2010 season; c. Sample collected between Apr. and Jun. of 2009 during 2008-2009 season and from Apr. to Jun. 2010 during 2009-2010 season; d. B/Florida/4/2006 (B/Yamagata lineage) for 2008-09 season and B/Brisbane/60/2008 (B/Victoria lineage) for 2009-2010 season; e. Homologous lineage strain: B/Taiwan/29/2008 (B/Yamagata lineage) in 2008-2009 influenza season and B/Taiwan/5908/2009 (B/Victoria lineage) in 2009-2010 influenza season; f. Heterologous lineage strain: B/Taiwan/5908/2009 in 2008-2009 influenza season and B/Taiwan/29/2008 in 2009-2010 influenza season.

To understand if the sero-protection rate post-vaccination was affected by the baseline pre-vaccination titre, the pre-vaccination sera during the 2009-10 season were obtained. For the 2009/10 B/Victoria vaccine antigen, the sero-protection rate in vaccinated subjects were $44.8 \%$, which was significantly lower than those in unvaccinated subjects with $82 \%(\mathrm{p}<0.05)$ (Table 2). Although the sero-protection rates against the vaccine and heterologous wild-type strains before receiving TIV were equivalently low in the vaccinated group, children under age 9 receiving one dose of 2009/10 TIV also demonstrated significantly higher sero-protection rate of 69\% 
against the homologous vaccine antigen from the same $\mathrm{B} /$ Victoria lineage than that of $27.6 \%$ against the heterologous wild-type antigen from the other $\mathrm{B} /$ Yamagata lineage. However, no differences in the sero-protection rate were observed between different lineage antigens in children $\geq 9$ years and the sera obtained during postseason in both season years.

\subsection{Fold Increase of HI before and Post-Vaccination}

Since the sero-protection rate post-vaccination was dependent on the pre-vaccination antibody titre, the change in antibody titre from before and after vaccination was further compared by GMTR to provide information on the vaccination effect. Post-vaccination with one dose of 2009/10 TIV in children under age 9 in years induced significantly higher immunological response to the homologous antigens from B/Victoria lineage with GMTR of 1.82 and 1.13 in vaccinated subjects, compared to the un-vaccinated subjects with GMTR of 0.57 and 0.68 for both strains, B/Brisbane/60/2008 and B/Taiwan/5908/2009, respectively $(\mathrm{p}<0.05)$ (Figure 2). However, a contradicting result of the antibody response to the heterologous $\mathrm{B} /$ Yamagata lineage antigen was observed with GMTR 0.73 in the vaccinated group, compared to 1.07 in the unvaccinated group with statistical significance (p $<0.05$ ). No difference in GMTR post-2009-10 TIV vaccination in children $\geq 9$ years old was observed.

\section{Discussion}

In the current study, we followed school-aged children with a well-defined vaccination history to assess subsequent immunological responses to TIV containing the alternate B/Lineage. Our results revealed that children under the age of 9 years in years receiving TIV showed higher HI titres and sero-protection rates of homologous strains from the same lineage than those of heterologous strains from the other lineage while no differences were observed for children in the older age group ( $\geq 9$ years old). Since 2008 , the ACIP recommends annual influenza vaccination for all children of 6 months to 18 years old, including all school-aged children [17]. The results shown in our study provided important information for countries which are planning, or currently implementing the TIV immunization programme in school-aged children.

Influenza B viruses currently circulating worldwide are of two distinct evolutionary hemagglutinin lineages, B/Yamagata and B/Victoria [13] [27] [28]. The distinct antigenicity between B/Yamagata and B/Victoria lineages has also been established based on antisera produced in ferrets, which were found to be highly specific against the homologous lineage with little or no cross-reactivity, suggesting a remarkable antigenic difference between these two lineages [7] [29] [30]. The results were further supported by the lack of cross-reactivity and neutralization activity observed in immunologically naïve volunteers [16]. Since the current trivalent influenza vaccines contain only one $B$ vaccine antigen, the mismatch between vaccine and wild-type antigens and the concurrent appearance of influenza B virus of both lineages during the same winter season are of major concern [14]. The question has been raised as to whether both lineages of B antigen should be represented in the annual influenza vaccine formulation. In this study, we examined whether immunization with a B viral antigen of one lineage is sufficient for producing antibodies with cross-reactivity against both lineages of B viruses. Our results confirmed that children receiving TIV showed higher HI titres and sero-protection rates of homologous strains from the same lineage than those of heterologous strains from the other lineage-either post-vaccination or post-influenza season. The need for the quadrivalent TIV requires further consideration.

Influenza B virus infections and immunizations often produce relatively low HI titres. This effect is not only the result of strain variability but also the method of measuring HI antibodies. Previous studies suggested a more sensitive but less specific assay when applying antigens from influenza B viruses after disruption by treatment with an organic solvent such as ether [31] [32]. The HI titres obtained with antigens after ether or detergent disruption correlate extremely well with neutralization antibody titres, and the HI titres at dilutions exceeding 1:32 after immunization with detergent-disrupted antigens can be associated with protection against illness and infection [31] [33]. In our study, sero-protection rates in subjects after immunization in 2008-09 and 2009-10 seasons were shown to be only $69 \%$ and $64 \%$ by measuring HI with non-disrupting B viruses as depicted in Table 2 . Since the presence of a HI antibody is the best surrogate measure of protection and the desired outcome of immunization [34], the antibody responses detected by HI with non-detergent-disrupted antigens suggest that the sero-protection rates against influenza B virus strains can be under-estimated in our study subjects.

The lower antibody response to the heterologous B/Yamagata lineage antigen among the vaccinated subjects during 2009-10 season as shown above could be related to different priming histories as suggested by previous 


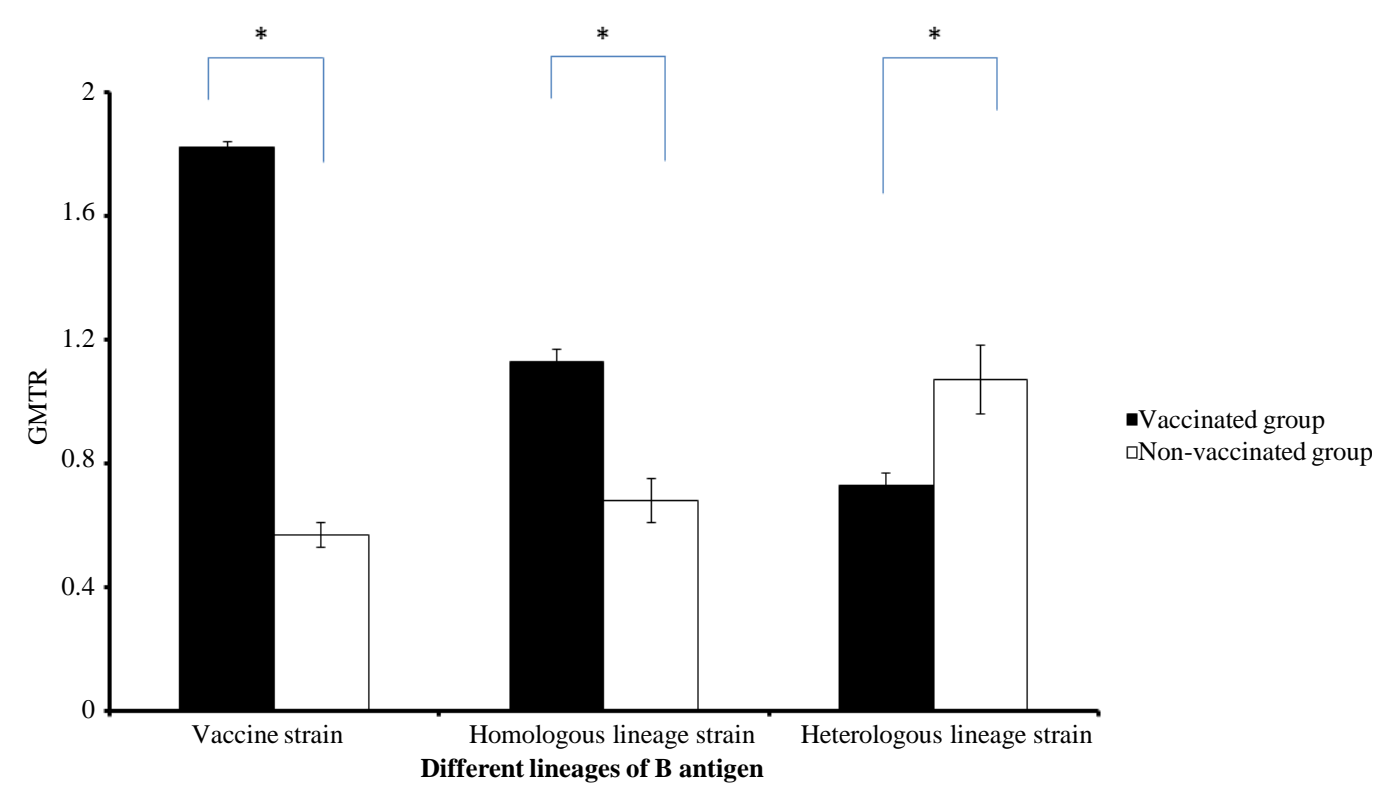

Figure 2. Comparison of geometric mean titre ratio (GMTR) measured by hemagglutination inhibition (HI) assay against homologous vaccine strain (B/Brisbane/60/2008-like, B/Victoria lineage), wild-type homologous lineage strain (B/Taiwan/5908/2009, B/Victoria lineage) and wild-type heterologous lineage strain (B/ Taiwan/29/2008, B/Yamagata lineage) between vaccinated and non-vaccinated children less than 9 years during 2009-10 influenza season. GMTR was calculated by dividing the post-vaccination GMT by the pre-vaccination baseline GMT. ${ }^{*}$ Indicated the statistical significance with $\mathrm{p}<0.05$.

studies [16] [35] [36]. Our study also attempted to understand whether priming with one dose of a specific influenza B/Lineage provided children with an adequate boost by a single dose of the other lineage in the consecutive year; the subjects were further divided into four groups: 1) receiving both lineages of B component of TIV in 2008/09 and 2009/10 influenza seasons; 2) receiving TIV containing B/Yamagata lineage only in 2008/09 but not receiving TIV containing B/Victoria lineage in 2009/10; 3) not receiving TIV containing B/Yamagata lineage only in 2008/09 but receiving TIV containing B/Victoria lineage in 2009/10; 4) not receiving TIV in both 2009/09 and 2009/10 influenza seasons. Although the sample size was small, the results revealed that receiving vaccination of both lineages in two consecutive years showed a significantly higher GMT of HI (107.67; 95\% CI: 72.5 - 144.9) against the vaccine strain post vaccination compared with children having no vaccination (35.54; 95\% CI: 24.9 - 51.5) for children under the age of 9 years, with statistical significance $(p<0.05)$ (data not shown). The significant differences between previous and the current studies included age of the study subjects and a 2-dose schedule was administered and repeated during the season that influenza B/Lineage changed. Evaluation of cohorts of children with a defined history of prior influenza B/Lineage prime-boost experiences across additional seasons will therefore be important for confirming these results and understanding their clinical relevance.

The main limitation of our study relates to the small sample sizes among subsets of the participants receiving alternating TIV, which reduced both the statistical power and precision. Furthermore, the sample bias could not be excluded since the current study design was non-randomized and different exposure experiences between vaccinated and unvaccinated groups might confound the results. Although influenza B was not a major contributor to virus circulation in Taiwan during the span of these sequential studies as shown in Figure 1, low level transmission in the community was still possible even though the $\mathrm{HI}$ titres in post-season sera were similar between vaccinated and non-vaccinated groups. Particularly, children are usually asymptomatic once infected by influenza viruses, and are therefore considered the primary community spreaders of the viruses [19] [37] [38]. At baseline antibody titres before vaccination during 2009-10 season (Table 2), a lower level of GMT and seroprotection rate for children under the age of 9 years in vaccinated group than that in un-vaccinated group was observed, which suggested asymptomatic infections among un-vaccinated subjects. Finally, we also cannot rule out the observations in this study could be particular to the specific influenza B strains and further evaluation in additional seasons is therefore warranted. 
The burden of influenza B infection falls mostly on children although the overall mortality rates associated with influenza B infection were less than those of A/H3N2, but greater than those of A/H1N1 [39]-[41]. In this study, the significantly higher HI titres and sero-protection rates of homologous strains from the same lineage than those of heterologous strains from the other lineage were showed among children under the age of 9 years in years after receiving TIV, while no differences were observed for children in the older age group ( $>9$ years old). Our finding provides both practical and scientific implications for the evaluation of influenza immunization programmes in school-aged children with more diverse exposures and antibody population of immunologic experience to both influenza B lineages.

\section{Acknowledgements}

This study was supported by National Science Council of Taiwan (NSC 97-2118-M-039-004) and China Medical University, Taiwan (CMU 97 323). The funders had no role in study design, data collection and analysis, decision to publish, or preparation of the manuscript. We declare that we have no conflict of interest.

\section{Declaration of Interest}

None.

\section{Financial Support}

This study was supported by National Science Council of Taiwan (NSC 97-2118-M-039-004) and China Medical University, Taiwan (CMU 97 323). The funders had no role in study design, data collection and analysis, decision to publish, or preparation of the manuscript.

\section{References}

[1] Cox, N.J. and Subbarao, K. (2005) Global Epidemiology of Influenza: Past and Present. Annual Review of Medicine, 51, 407-421. http://dx.doi.org/10.1146/annurev.med.51.1.407

[2] Boni, M.F. (2008) Vaccination and Antigenic Drift in Influenza. Vaccine, 26, C8-C14. http://dx.doi.org/10.1016/j.vaccine.2008.04.011

[3] Couch, R.B. (2000) Prevention and Treatment of Influenza. New England Journal of Medicine, 343, 1778-1787. http://dx.doi.org/10.1056/NEJM200012143432407

[4] Carrat, F. and Flahault, A. (2007) Influenza Vaccine: The Challenge of Antigenic Drift. Vaccine, 25, 6852-6862. http://dx.doi.org/10.1016/j.vaccine.2007.07.027

[5] Shaw, M., et al. (2002) Reappearance and Global Spread of Variants of Influenza B/Victoria/2/87 Lineage Viruses in the 2000-2001 and 2001-2002 Seasons. Virology, 303, 1-8. http://dx.doi.org/10.1006/viro.2002.1719

[6] Chi, X., et al. (2005) Detection and Characterization of New Influenza B Virus Variants in 2002. Journal of Clinical Microbiology, 43, 2345-2349. http://dx.doi.org/10.1128/JCM.43.5.2345-2349.2005

[7] Puzelli, S., et al. (2004) Changes in the Hemagglutinins and Neuraminidases of Human Influenza B Viruses Isolated in Italy during the 2001-02, 2002-03, and 2003-04 Seasons. Journal of Medical Virology, 74, 629-640. http://dx.doi.org/10.1002/jmv.20225

[8] Lin, J., et al. (2007) Characterization of the Epidemic Influenza B Viruses Isolated during 2004-2005 Season in Taiwan. Virus Research, 124, 204-211. http://dx.doi.org/10.1016/j.virusres.2006.11.005

[9] Jian, J., et al. (2008) Genetic and Epidemiological Analysis of Influenza Virus Epidemics in Taiwan during 2003 to 2006. Journal of Clinical Microbiology, 46, 1426-1434. http://dx.doi.org/10.1128/JCM.01560-07

[10] Shih, S., et al. (2005) Laboratory-Based Surveillance and Molecular Epidemiology of Influenza Virus in Taiwan. Journal of Clinical Microbiology, 43, 1651-1661. http://dx.doi.org/10.1128/JCM.43.4.1651-1661.2005

[11] Jian, J.-W., Lai, C.-T., Kuo, C.-Y., Kuo, S.H.S., Hsu, L.-C., Chen, P.-J., et al. (2008) Genetic Analysis and Evaluation of the Reassortment of Influenza B Viruses Isolated in Taiwan during the 2004-2005 and 2006-2007 Epidemics. Virus Research, 131, 243-249. http://dx.doi.org/10.1016/j.virusres.2007.09.014

[12] Tsai, H.-P., Wang, H.-C., Kiang, D., Huang, S.-W., Kuo, P.-H., Liu, C.-C., et al. (2006) Increasing Appearance of Reassortant Influenza B Virus in Taiwan from 2002 to 2005. Journal of Clinical Microbiology, 44, 2705-2713. http://dx.doi.org/10.1128/JCM.02694-05

[13] Belshe, R.B. (2010) The Need for Quadrivalent Vaccine against Seasonal Influenza. Vaccine, 28, D45-D53. 
http://dx.doi.org/10.1016/j.vaccine.2010.08.028

[14] Kanegae, Y., Sugita, S., Endo, A., Ishida, M., Senya, S., Osako, K., et al. (1990) Evolutionary Pattern of the Hemagglutinin Gene of Influenza B Viruses Isolated in Japan: Cocirculating Lineages in the Same Epidemic Season. Journal of Virology, 64, 2860-2865.

[15] Rota, P.A., Wallis, T.R., Harmon, M.W., Rota, J.S., Kendal, A.P. and Nerome, K. (1990) Cocirculation of Two Distinct Evolutionary Lineages of Influenza Type B Virus since 1983. Virology, 175, 59-68. http://dx.doi.org/10.1016/0042-6822(90)90186-U

[16] Levandowski, R.A., Gross, P.A., Weksler, M., Staton, E., Williams, M.S. and Bonelli, J. (1991) Cross-Reactive Antibodies Induced by a Monovalent Influenza B Virus Vaccine. Journal of Clinical Microbiology, 29, 1530-1532.

[17] Fiore, A.E., Shay, D.K., Broder, K., Iskander, J.K., Uyeki, T.M., Mootrey, G., et al. (2008) Prevention and Control of Influenza: Recommendations of the Advisory Committee on Immunization Practices (ACIP), 2008. Morbidity and Mortality Weekly Report Recommendations and Report, 57, 1-60.

[18] Hurwitz, E.S., Haber, M., Chang, A., Shope, T., Teo, S., Ginsberg, M., et al. (2000) Effectiveness of Influenza Vaccination of Day Care Children in Reducing Influenza-Related Morbidity among Household Contacts. The Journal of the American Medical Association, 284, 1677-1682. http://dx.doi.org/10.1001/jama.284.13.1677

[19] Reichert, T.A., Sugaya, N., Fedson, D.S., Glezen, W.P., Simonsen, L. and Tashiro, M. (2001) The Japanese Experience with Vaccinating Schoolchildren against Influenza. New England Journal of Medicine, 344, 889-896. http://dx.doi.org/10.1056/NEJM200103223441204

[20] Davis, M.M., King Jr., J.C., Moag, L., Cummings, G. and Magder, L.S. (2008) Countywide School-Based Influenza Immunization: Direct and Indirect Impact on Student Absenteeism. Pediatrics, 122, e260-e265. http://dx.doi.org/10.1542/peds.2007-2963

[21] Su, W.-J., Shao, P.-L., Liu, M.-T., Liu, D.-P., Huang, K.-C., Chang, L.-Y., et al. (2010) Low Seroprotection against Preseasonal Influenza Local Strains in Children Might Predict the Upcoming Epidemic Influenza Strains. Clinical Infectious Diseases, 51, 171-176. http://dx.doi.org/10.1086/653532

[22] Bodewes, R., de Mutsert, G., van der Klis, F.R.M., Ventresca, M., Wilks, S., Smith, D.J., et al. (2011) Prevalence of Antibodies against Seasonal Influenza A and B Viruses in Children in Netherlands. Clinical Vaccine and Immunology, 18, 469-476. http://dx.doi.org/10.1128/CVI.00396-10

[23] Rowe, T., Abernathy, R.A., Hu-Primmer, J., Thompson, W.W., Lu, X.H., Lim, W., et al. (1999) Detection of Antibody to Avian Influenza A (H5N1) Virus in Human Serum by Using a Combination of Serologic Assays. Journal of Clinical Microbiology, 37, 937-943.

[24] World Health Organization (2002) WHO Manual on Animal Influenza Diagnosis and Surveillance. WHO/CDS/CSR/ NCS/20025.

[25] Yang, J.-R., Lin, C.-H., Chen, C.-J., Liu, J.-L., Huang, Y.-P., Kuo, C.-Y., et al. (2010) A New Antigenic Variant of Human Influenza A (H3N2) Virus Isolated from Airport and Community Surveillance in Taiwan in Early 2009. Virus Research, 151, 33-38. http://dx.doi.org/10.1016/j.virusres.2010.03.011

[26] Yang, J.-R., Lo, J., Liu, J.-L., Lin, C.-H., Ho, Y.-L., Chen, C.-J., et al. (2009) Rapid SYBR Green I and Modified Probe Real-Time Reverse Transcription-PCR Assays Identify Influenza H1N1 Viruses and Distinguish between Pandemic and Seasonal Strains. Journal of Clinical Microbiology, 47, 3714-3716. http://dx.doi.org/10.1128/JCM.01646-09

[27] McCullers, J.A., Saito, T. and Iverson, A.R. (2004) Multiple Genotypes of Influenza B Virus Circulated between 1979 and 2003. Journal of Virology, 78, 12817-12828. http://dx.doi.org/10.1128/JVI.78.23.12817-12828.2004

[28] Hite, L.K., Glezen, W.P., Demmler, G.J. and Munoz, F.M. (2007) Medically Attended Pediatric Influenza during the Resurgence of the Victoria Lineage of Influenza B Virus. International Journal of Infectious Diseases, 11, 40-47. http://dx.doi.org/10.1016/j.ijid.2005.10.008

[29] Rota, P.A., Hemphill, M.L., Whistler, T., Regnery, H.L. and Kendal, A.P. (1992) Antigenic and Genetic Characterization of the Haemagglutinins of Recent Cocirculating Strains of Influenza B Virus. Journal of General Virology, 73, 2737-2742. http://dx.doi.org/10.1099/0022-1317-73-10-2737

[30] Robertson, J.S., Bootman, J.S., Nicolson, C., Major, D., Robertson, E.W. and Wood, J.M. (1990) The Hemagglutinin of Influenza B Virus Present in Clinical Material Is a Single Species Identical to That of Mammalian Cell-Grown Virus. Virology, 179, 35-40. http://dx.doi.org/10.1016/0042-6822(90)90270-2

[31] Kendal, A.P. and Cate, T.R. (1983) Increased Sensitivity and Reduced Specificity of Hemagglutination Inhibition Tests with Ether-Treated Influenza B/Singapore/222/79. Journal of Clinical Microbiology, 18, 930-934.

[32] Monto, A.S. and Maassab, H.F. (1981) Ether Treatment of Type B Influenza Virus Antigen for the Hemagglutination Inhibition Test. Journal of Clinical Microbiology, 13, 54-47.

[33] Frank, A.L., Taber, L.H., Glezen, W.P., Geyer, E.A., McIlwain, S. and Paredes, A. (1983) Influenza B Virus Infections 
in the Community and the Family: The Epidemics of 1976-1977 and 1979-1980 in Houston, Texas. American Journal of Epidemiology, 118, 313-325.

[34] Hannoun, C., Megas, F. and Piercy, J. (2004) Immunogenicity and Protective Efficacy of Influenza Vaccination. Virus Research, 103, 133-138. http://dx.doi.org/10.1016/j.virusres.2004.02.025

[35] Levandowski, R.A., Regnery, H.L., Staton, E., Burgess, B.G., Williams, M.S. and Groothuis, J.R. (1991) Antibody Responses to Influenza B Viruses in Immunologically Unprimed Children. Pediatrics, 88, 1031-1036.

[36] Englund, J.A., Walter, E.B., Gbadebo, A., Monto, A.S., Zhu, Y. and Neuzil, K.M. (2006) Immunization with Trivalent Inactivated Influenza Vaccine in Partially Immunized Toddlers. Pediatrics, 118, e579-e585. http://dx.doi.org/10.1542/peds.2006-0201

[37] Reichert, T. (2002) The Japanese Program of Vaccination of Schoolchildren against Influenza: Implications for Control of the Disease. Seminars in Pediatric Infectious Diseases, 13, 104-111. http://dx.doi.org/10.1053/spid.2002.122997

[38] King, J.C., Stoddard, J.J., Gaglani, M.J., Moore, K.A., Magder, L., McClure, E., et al. (2006) Effectiveness of SchoolBased Influenza Vaccination. New England Journal of Medicine, 355, 2523-2532. http://dx.doi.org/10.1056/NEJMoa055414

[39] Li, W.-C., Shih, S.-R., Huang, Y.-C., Chen, G.-W., Chang, S.-C., Hsiao, M.-J., et al. (2008) Clinical and Genetic Characterization of Severe Influenza B-Associated Diseases during an Outbreak in Taiwan. Journal of Clinical Virology, 42, 45-51. http://dx.doi.org/10.1016/j.jcv.2007.11.026

[40] Thompson, W.W., Shay, D.K., Weintraub, E., Brammer, L., Cox, N., Anderson, L.J. and Fukuda, K. (2003) Mortality Associated with Influenza and Respiratory Syncytial Virus in the United States. The Journal of the American Medical Association, 289, 179-186. http://dx.doi.org/10.1001/jama.289.2.179

[41] Glezen, W.P., Couch, R.B., Taber, L.H., Paredes, A., Allison, J.E., Frank, A.L. and Aldridge, C. (1980) Epidemiologic Observations of Influenza B Virus Infections in Houston, Texas, 1976-1977. American Journal of Epidemiology, 111, 13-22. 
Scientific Research Publishing (SCIRP) is one of the largest Open Access journal publishers. It is currently publishing more than 200 open access, online, peer-reviewed journals covering a wide range of academic disciplines. SCIRP serves the worldwide academic communities and contributes to the progress and application of science with its publication.

Other selected journals from SCIRP are listed as below. Submit your manuscript to us via either submit@scirp.org or Online Submission Portal.
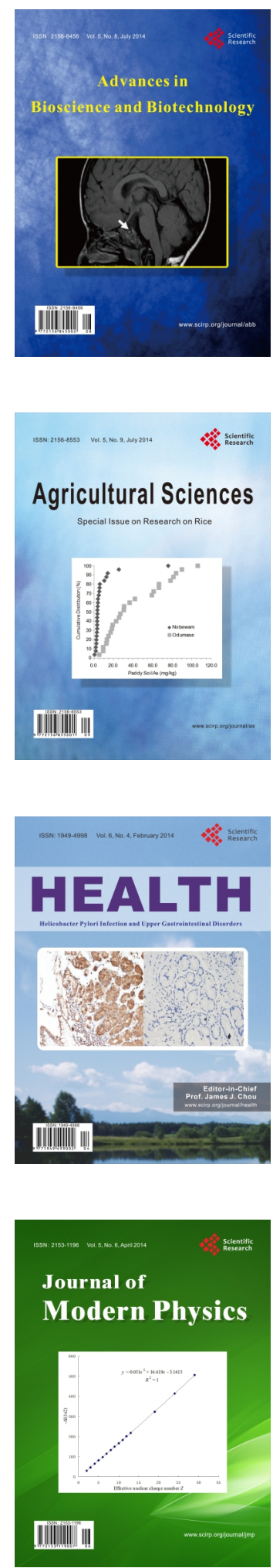
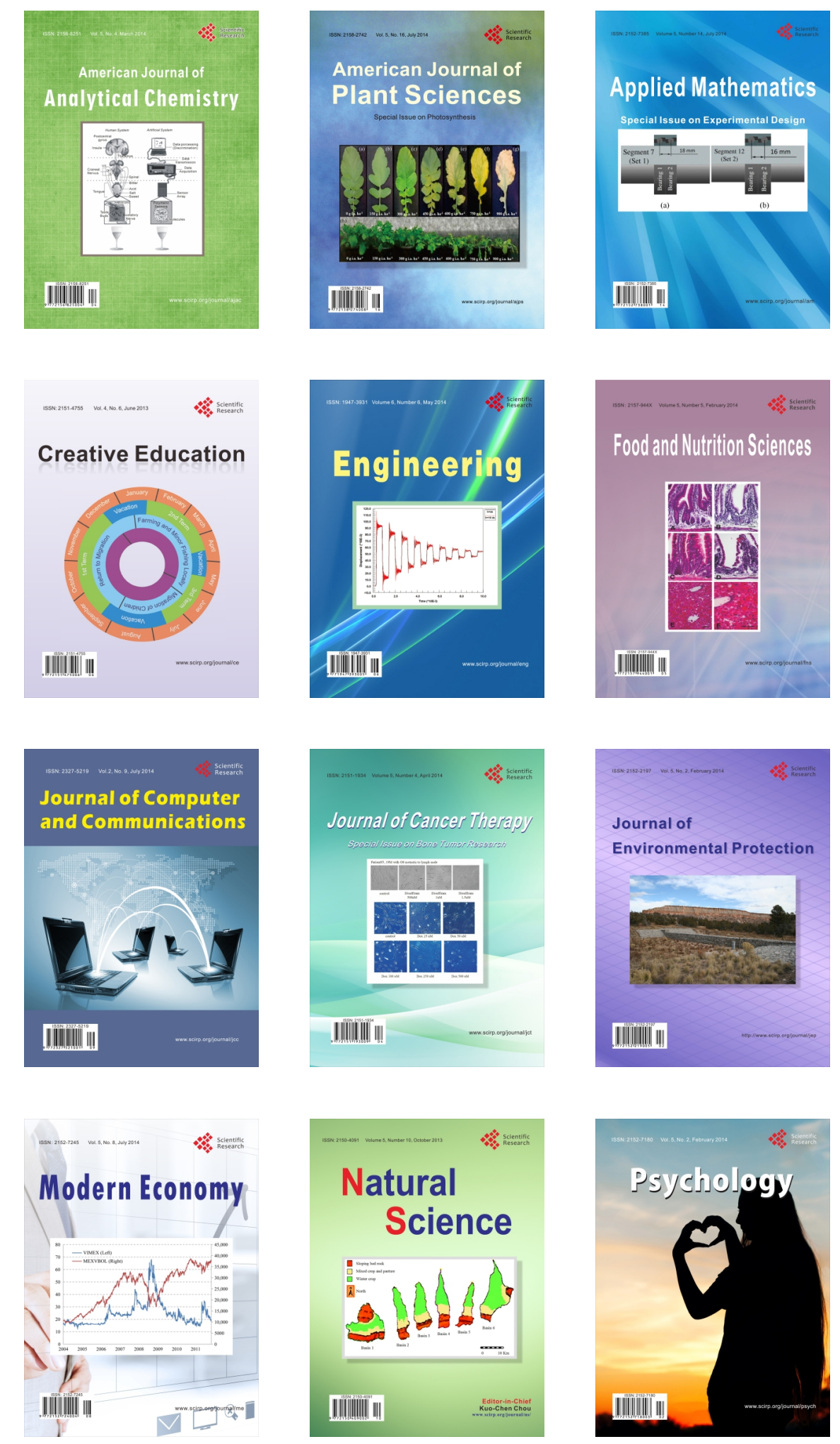